\title{
Tägliche Mesalazin-Einmaldosis überlegen
}

Die remissionserhaltende Wirkung der seit Jahrzehnten bei Colitis Ulcerosa (CU) eingesetzten Aminosalizylate verschlechtert sich häufig durch nachlassende Einnahmedisziplin. Mit einer hochdosierten Mesalazin-Einzeltagesdosis können Compliance und Therapieerfolg deutlich verbessert werden.

Die aktuellen europäischen und deutschen Leitlinien für die Behandlung der CU bestätigen die Wirksamkeit sowie Sicherheit von Aminosalizylaten und empfehlen Mesalazin als Erstlinientherapie. Da nahezu alle CU-Patienten ein Rezidiv bekommen, wenn die Therapie zu früh abgebrochen wird, ist meist eine Dauertherapie erforderlich, betonte Privatdozent Dr. Niels Teich, Leipzig. Dabei sollten Aminosaliylate primär als remissionserhaltende Therapie eingesetzt werden, wenn ein Ansprechen auf Aminosalizylate oder Steroide besteht, die Kombination oraler und rektaler Aminosalizylate gilt als Zweitlinien-Erhaltungstherapie. Eine remissionserhaltende Therapie mit Aminosalyzylaten sollte bei Effektivität dann mindestens zwei Jahre durchgeführt werden. Dadurch ergeben sich aber fast immer Probleme bei der Compliance, erklärte Teich, denn nach seiner Erfahrung sei es bei Patienten in Remission völlig normal, wenn sie mindestens einmal pro Woche ihr Mesalazinpräparat vergessen. Durch Problemkonstellationen wie Vollzeitbeschäftigung, dreimal tägliche Einnahme, viele Tabletten, viel Komedikation, Linksseitenkolitis oder letzte Koloskopie vor mehr als zwei Jahren sinke die Mesalazin-Compliance auf unter $70 \%$.

Mit der täglichen Einmalgabe von Mesalazin werde eindeutig die Compliance aber wohl auch die Wirksamkeit gesteigert, wie verschiedene klinische Studien gezeigt hätten. Angelegt waren diese Stu- dien auf Compliance, aber wiederholt nachgewiesen war die Wirkung der Einmalgabe besser, betonte Teich. Auch bei aktiver CU war die Einmaldosis der Mehrfachgabe von Mesalazin nicht unterlegen. In einer randomisierten klinischen Studie erhielten 206 Patienten mit milder bis mittelschwerer CU über acht Wochen täglich $4 \mathrm{~g}$ (2 g Sachets) retardiertes zeitabhängig freisetzendes Mesalazin (Pentasa ${ }^{\circledR}$ ) entweder nur morgens (1-0-0) oder morgens und abends (1-0-1) plus $1 \mathrm{~g}$ Klistier (vier Wochen) [Fluorie B et al. Aliment Pharmacol Ther 2013; 37: 767 - 75]. Bei der endoskopischen und klinischen Remission nach acht Wochen (primärer Endpunkt) hatten beide Einnahmeregimes eine statistisch vergleichbar gute Wirkung $(52,1$ vs. $41,8 \%, p=0,14)$. Die Mukosaheilung wurde mit der Einmaldosis sogar signifikant häufiger als mit der Zweimalgabe erreicht $(87,5$ vs. $71,1 \%, p=0,007)$, resümierte Teich.

Dr. Thomas Riedel

Satellitensymposium „Mikrobiom und gastrointestinale Erkrankungen", Viszeralmedizin 2017, 15.9.2017, Dresden; Veranstalter: Ferring

\section{Pembrolizumab-Monotherapie bei stark vorbehandelten Patienten}

Magenkrebs ist weltweit die fünfthäufigste Krebsform und die dritthäufigste Ursache krebsbedingter Todesfälle. Schätzungen zufolge liegt die Neuerkrankungsrate für Magenkrebs im Jahr 2020 allein in Deutschland bei mehr als 14.000 Menschen.

In der Phase-II-Studie KEYNOTE-059 wird der PD-1(Programmed Cell Death 1 Protein)-Inhibitor Pembrolizumab als Monotherapie bei vorbehandelten Patienten mit fortgeschrittenem Adenokarzinom des Magens oder des gastroösophagealen Übergangs untersucht. Bei $\mathrm{Pa}$ tienten, die bereits mindestens zwei Chemotherapielinien erhalten haben (Kohorte 1), wird Pembrolizumab als Monotherapie untersucht (200 mg alle drei Wochen für bis zu 24 Monate). Die primären Endpunkte sind die ORR (Overall Response Rate), beurteilt durch einen verblindeten, unabhängigen, zentralen, radiografischen Prüfprozess (BICRC) ge- mäß RECIST v1.1D, sowie die Sicherheit. Sekundäre Endpunkte sind die Ansprechdauer und das Gesamtüberleben sowie progressionsfreies Überleben. Es wurde eine weitere Auswertung basierend auf der Tumor-PD-L1-Expression durchgeführt. Hierbei war die positive Tumor-PD-L1-Expression als kombinierter positiver Score (CPS) der Tumor- und Immunzellen von $\geq 1 \%$ definiert.

Das Unternehmen MSD gab kürzlich die Ergebnisse zur Kohorte 1 bekannt, die eine Gesamtansprechrate (ORR) von 11,6\% (95\%-KI: 8,0-16,1) bei Patienten unter Pembrolizumab zeigen, die bereits mindestens zwei Therapielinien erhalten hatten. Die Gesamtansprechraten bei Patienten mit positiver Tumor-PD-L1Expression waren dabei höher (15,5\%). Diese Ergebnisse wurden auf der diesjährigen 53. Jahrestagung der American Society of Clinical Oncology in Chicago vorgestellt (Abstract \#4003). Sie unter- stützen die Zulassungserweiterung für Pembrolizumab, die von MSD eingereicht wurde und sich derzeit im beschleunigten Prüfverfahren befindet. Mit diesem Lizenzantrag wird die Zulassung des PD-1-Inhibitors zur Behandlung von Patienten mit rezidivierendem oder fortgeschrittenem Adenokarzinom des Magens oder des gastroösophagealen Übergangs, die bereits mindestens zwei Chemotherapielinien erhalten haben, angestrebt.

KEYNOTE-059 ist aktuell nur in den USA zulassungsrelevant - in Deutschland und der Europäischen Union ist dies zurzeit noch nicht der Fall.

„Magenkrebs im fortgeschrittenen Stadium ist schwer behandelbar, daher besteht für diese Patienten ein erheblicher Bedarf an neuen Behandlungsoptionen“, sagte Dr. Charles S. Fuchs, MPH, Studienleiter und Direktor des Yale Cancer Centers. „Die Ergebnisse der KEYNOTE-059 zeigen bedeutende Ansprechraten unter Pembrolizumab bei stark vorbehandelten Patienten, die unter den bisherigen Therapieoptionen nicht erfolgreich behandelt werden konnten." red

Nach Informationen von MSD 